\title{
Die Lampen-Moosflora der Beatushöhle und deren Vergleich mit anderen europäischen Höhlen
}

\author{
Von R. BERnASGONI ${ }^{1}$
}

\section{Frühere Arbeiten über die Lampen-Moosflora der Beatushöhle}

Der Raum um elektrische Glühbirnen in Schauhöhlen stellt einen besonderen Biotop für eine spezielle Florula dar (Vogellehner 1963b). Bereits 1904 wurde die Beatushöhle am Thunersee mit elektrischer Beleuchtung ausgerüstet (Stammler 1904), und 1924 berichtet Lüdy als erster über ihre Lampen-Florula, wobei er eine Farnart, eine Algenart sowie 8 Moosarten erwähnt. Es sind dies: Bryum sp.; Encalypta contorta Wulf.; Eurynchium schwartzi Turn. f. schistostegioides Gams; Fissidens decipiens DeNot; Hymenosytilium curvirostre Ehrb.; Isopterygium depressum Br. f. cavernarum Gams; Tortella tortuosa L. f. tenuis; Tortula muralis L. (Lüdy 1904; Morton, Gams 1925).

Die anatomischen Veränderungen dieser Höhlenmoose der Beatushöhle untersuchte Elise Hofmann (in: Morton 1927); sie fand dabei regelmäßige Reduktion der Beblätterung, der Blattzähnchen, des assimilierenden Gewebes, ferner eine Verlängerung des Stämmchens; dies alles wurde als Ökonomieprinzip gedeutet. Im folgenden sind diese Untersuchungen an 5 Beatushöhlenmoosen tabellarisch zusammengestellt und mit Normalformen verglichen.

Tabelle 1

Anatomische Veränderungen der Beatushöhlenmoose

\begin{tabular}{lcccc}
\hline Art & $\begin{array}{c}\text { Stämmchen- } \\
\text { querschnitt } \\
\mu\end{array}$ & $\begin{array}{c}\text { Länge } \times \text { Breite } \\
\text { der Epidermiszellen } \\
\mu\end{array}$ & Blattdicke & Rippendicke \\
\hline Tortula muralis & & & $\mu$ & $\mu$ \\
Höhlenform & 49 & $19 \times 9,8$ & 29 & 66 \\
Normalform & 52 & $33 \times 16,9$ & 32 & 70
\end{tabular}

1) Société Suisse de Spéléologie, Bern, Mergartenstr. 13, Suisse. 
Tabelle 1 (Fortsetzung)

\begin{tabular}{lcccc}
\hline Art & $\begin{array}{c}\text { Stämmchen- } \\
\text { querschnitt }\end{array}$ & $\begin{array}{c}\text { Länge } \times \text { Breite } \\
\text { der Epidermiszellen }\end{array}$ & Blattdicke & Rippendicke \\
\end{tabular}

\begin{tabular}{|c|c|c|c|c|}
\hline & $\mu$ & $\mu$ & $\mu$ & $\mu$ \\
\hline \multicolumn{5}{|c|}{ Tortella tortuosa } \\
\hline Höhlenform & 166 & $33 \times 9,8$ & 11 & 33 \\
\hline Normalform & 166 & $49 \times 8,6$ & 16 & 83 \\
\hline \multicolumn{5}{|c|}{ Encalypta contorta } \\
\hline Höhlenform & 247 & $33 \times 16,0$ & 23 & 71 \\
\hline Normalform & 249 & $33 \times 13,8$ & 24 & 61 \\
\hline \multicolumn{5}{|c|}{ Eurynchium schwartzi f. schistostegoides } \\
\hline Höhlenform & 132 & $74 \times 16,0$ & 16 & 43 \\
\hline Normalform & 265 & $91 \times 8,0$ & 9 & 41 \\
\hline \multicolumn{5}{|c|}{ Isopterygium depressum } \\
\hline Höhlenform & 182 & $66 \times 6,0$ & 13 & 13 \\
\hline Normalform & 265 & $96 \times 16,0$ & 16 & 16 \\
\hline
\end{tabular}

\section{Eigene Untersuchungen}

\subsection{Die untersuchten Fundorte}

Der touristisch ausgebaute Teil der Beatushöhle beträgt etwa $700 \mathrm{~m}$ und ist mit zahlreichen Lampen und Scheinwerfern ausgerüstet. Nicht jede Lampenumgebung besitzt eine gleichreiche Florula, einige weisen nur eine spärliche Algenflorula auf. Man beschränkte sich schließlich auf folgende 8 Fundorte, die besonders interessante oder reichhaltige Moosrasen aufwiesen. Von jedem Fundort bestimmte man die Temperatur, die relative Feuchtigkeit und die Beleuchtungsstärke. Zur Messung der Temperatur und der relativen Feuchtigkeit benützte man ein Psychrometer, zur Messung der Beleuchtungsstärke ein Luxmeter. Die Werte beziehen sich auf das Zentrum des Rasens.

Tabelle 2

Die untersuchten Fundorte

\begin{tabular}{|c|c|c|c|c|c|}
\hline & $\begin{array}{c}\text { Entfer- } \\
\text { nung vom } \\
\text { Eingang } \\
\text { m }\end{array}$ & $\begin{array}{l}\text { Lampe } \\
\text { W }\end{array}$ & $\begin{array}{c}\text { Tempe- } \\
\text { ratur } \\
\mathrm{G}^{\circ} \\
\end{array}$ & $\begin{array}{c}\text { Relative } \\
\text { Feuch- } \\
\text { tigkeit } \\
\% \\
\%\end{array}$ & $\begin{array}{c}\text { Beleuch- } \\
\text { tungs- } \\
\text { stärke } \\
\text { (Lux) }\end{array}$ \\
\hline Erste Quelle & 60 & 200 & 9,6 & 92 & 1100 \\
\hline Unterhalb steiler Treppe & 175 & 100 & 9,0 & 94 & 400 \\
\hline Beim alten Schalter & 195 & 60 & 10,2 & 95 & 360 \\
\hline Drei Schwestern (Gang) & 255 & 60 & 10,2 & 95 & $80-120$ \\
\hline Drei Schwestern (See) & 260 & 200 & 10,2 & 95 & 1100 \\
\hline Schlangengrotte & 675 & 150 & 11,8 & 87 & $<20-80$ \\
\hline Hexenkessel & 680 & 150 & 9,0 & 93 & 5000 \\
\hline Hades (Elephanten) & 685 & 60 & 9,0 & 93 & 7000 \\
\hline
\end{tabular}




\subsection{Die Moosflorula}

Die gesammelten Moose gelangten im frischen Zustand zur Bestimmung. Diese verdanke ich Herrn Dr. Ochsner, Muri AG.

Tabelle 3

Die gesammelten Moose

\begin{tabular}{ll}
\hline Fundort & Art \\
\hline Erste Quelle & Fissidens taxifolius f. cavernarum \\
Unterhalb steiler Treppe & Brachytecium salebrosum \\
& Eurynchium rusciforme \\
Beim alten Schalter & Eucladium verticillatum \\
Drei Schwestern (Gang) & Eucladium verticillatum f. cavernarum \\
Drei Schwestern (See) & Eucladium verticillatum f. tenuis \\
Schlangengrotte & Pohlia cruda \\
& Pellia epiphylla \\
Hexenkessel & Amblystegium juratzkanum \\
& Cratoneurum commutatum f. tenuis \\
& Eurynchium praelongum f. cavernarum \\
& Eurynchium praelongum typ. \\
& Bryum capillare \\
Hades (Elephanten) & Rhynchostegium murale \\
& Mniobrium albicans \\
& Fissidens taxifolius \\
& Eurynchium praelongum typ. \\
& Cratoneurum commutatum f. cavernarum
\end{tabular}

2.3. Einfluß des Höhlenklimas auf die Moosflorula

\subsubsection{Licht}

Das Lichtbedürfnis der Höhlenmoose ist meistens sehr gering (Morton, Gams 1925). Nach neueren Messungen (Vogellehner 1963a) dürfte eine Beleuchtungsstärke von 40 Lux die unterste Grenze für Moose sein; darunter wachsen nur noch Algen. Dieser Wert gilt z. B. für Fissidens taxifolius; bei anderen Arten (Eurynchium schwartzi, Cratoneurum commutatum) liegt sie bei 400 Lux. Vergleiche der Lichtmengen, welche den Moosen zur Verfügung stehen, zeigen zwischen einem extrem schattigen Außenstandort und einem optimalen Höhlenstandort praktisch keinen Unterschied: für Fissidens taxifolius wurden somit an diesen beiden Standorten rund 5 Kiloluxstunden gefunden. Die Beleuchtungsstärke um die Lampen nimmt mit zunehmender Entfernung von der Lampe nach folgendem Gesetz rasch $\mathrm{ab}$ :

$$
\text { Beleuchtungsstärke (Lux) }=\frac{\text { Lichtstärke (Lumen) }}{\text { Quadrat des Abstandes }\left(\mathrm{m}^{2}\right)}
$$


Die Lichtabnahme bewirkt entweder rhizoides Wachstum oder Umwandlung zu Höhlenformen und schließlich das totale Einstellen des Wachstums.

Der Bestand von Pohlia cruda weist z. B. nahe der Lampe einen grünen Gürtel, bei größerer Entfernung einen rhizoiden braunen Gürtel auf. Es wurden folgende Werte gemessen:

$$
\text { Tabelle } 4
$$

Der Moosbestand von Pohlia cruda

\begin{tabular}{lcc}
\hline Aussehen des Rasens & $\begin{array}{c}\text { Entfernung } \\
\text { von der Lampe } \\
\text { cm }\end{array}$ & $\begin{array}{c}\text { Beleuchtungs- } \\
\text { stärke } \\
\text { (Lux) }\end{array}$ \\
\hline Beginn des grünen Rasens & 20 & 3400 \\
Ende des grünen Rasens, & & \\
Beginn des rhizoiden Rasens & 50 & 1800 \\
Ende des rhizoiden Rasens & 150 & 140
\end{tabular}

Bei anderen Arten bilden sich bei abnehmender Beleuchtungsstärke Schatten- und schließlich Höhlenformen (Scialo- und Kryptomorphosen). So soll z. B. Isopterygium depressum bei $1 / 200$ bis ${ }^{1} / 300$ der Tageslichtintensität in seine f. cavernarum übergehen (Morton, Gams 1925).

Bei 3 Moosarten fanden wir folgende Reduzierungen:

Tabelle 5

Höhlenformen und Beleuchtungsstärken bei 3 Moosarten

\begin{tabular}{lrl}
\hline \multicolumn{1}{c}{ Moosart } & $\begin{array}{c}\text { Beleuch- } \\
\text { tungs- } \\
\text { stärke } \\
\text { (Lux) }\end{array}$ & \multicolumn{1}{c}{ Fundort } \\
\hline Eurynchium praelongum f. typ. & 7000 & Hades \\
& 5000 & Hexenkessel \\
Eurynchium praelongum f. cavernarum & 80 & Schlangengrotte \\
Cratoneurum commutatum f. cavernarum & 7000 & Hades \\
Cratoneurum commutatum f. tenuis & $<20$ & Schlangengrotte \\
Eucladium verticillatum f. typ. & 400 & unterhalb steiler Treppe \\
Eucladium verticillatum f. tenuis & 80 & Drei Schwestern \\
Eucladium verticillatum f. cavernarum & 120 & Drei Schwestern
\end{tabular}

Bei fast allen Moosarten, besonders aber bei Fissidens taxifolius, ist ein deutlicher Phototropismus festzustellen. Fissidens taxifolius (erste Quelle; Hexenkessel) richtet seine zweizeilig in einer Ebene liegenden Blättchen senkrecht gegen das Licht. Ein ebenfalls gegen das Licht 
gerichtetes Wachstum ist deutlich bei Eucladium verticillatum, Eurynchium schwartzi und Pohlia cruda zu beobachten. Das Wachstum von Amblystegium juratzkanum ist demgegenüber nicht phototropisch gerichtet.

\subsubsection{Temperatur}

Die Temperatur um die Lampen nimmt mit abnehmender Distanz zu. Bei Lampen mit 100-200 W ändert sich die Temperatur bei einer Entfernung von $20 \mathrm{~cm}$ kaum mehr; deshalb beginnt auch das Wachstum der Moosbestände in einer Entfernung von $20-30 \mathrm{~cm}$ von der Lampe. Beim Moosbestand von Pohlia cruda $(200 \mathrm{~W})$ wurden folgende Werte gemessen:

Tabelle 6

Der Moosbestand von Pohlia cruda

\begin{tabular}{ccc}
\hline Aussehen des Rasens & $\begin{array}{c}\text { Entfernung } \\
\text { von der Lampe } \\
\text { cm }\end{array}$ & $\begin{array}{c}\text { Temperatur } \\
\text { S })\end{array}$ \\
\hline Sterile Zone & 2,5 & 18,0 \\
& 5,0 & 15,2 \\
Beginn des grünen Rasens & 10,0 & 12,2 \\
& 15,0 & 10,1 \\
& 20,0 & 9,2 \\
& 30,0 & 9,0
\end{tabular}

\subsubsection{Feuchtigkeit}

Die relative Luftfeuchtigkeit beträgt bei den untersuchten Stellen 87-95\%. Änderungen durch das Heißwerden der Lampen konnten nicht festgestellt werden. Die hohe Luftfeuchtigkeit hat höchstens einen günstigen Einfluß auf das Mooswachstum.

\subsubsection{Substrat}

Sämtliche beobachteten Moosrasen wuchsen auf Höhlenlehm oder aus Felsspalten, die ebenfalls etwas Höhlenlehm enthielten.

\subsubsection{Fruchtkörperbildung}

Alle beobachteten Moose der Beatushöhle waren steril. Fruchtende Höhlenmoose sind übrigens nicht häufig beschrieben worden: so z. B. Amblystegium juratzkanum (Coûteau 1956; Koppe 1961); Seligeria doniana (Vogellehner 1963a); Cratoneurum commutatum (Vogellehner 1963a); Brachytecium velutinum (Koppe 1961); Leptobryum pyriforme (Boros 1964).

\footnotetext{
1) Gemessen $2 \mathrm{~cm}$ über dem Boden.
} 


\subsection{Charakterisierung der eingedrungenen Beatushöhlenmoose}

Unterteilt man die von Lüdy (1924) und von uns in der Beatushöhle gesammelten Moose in calcophile (kalkliebende), gesteinsindifferente, hygrophile (feuchtigkeitsliebende) und scialophile (schattenliebende), erhält man folgende prozentualen Zahlen (17 Arten):

\section{Tabelle 7}

Charakterisierung der Beatushöhlenmoose

\begin{tabular}{|c|c|c|}
\hline Substrat: & calcophile Moose & 6 Arten $=35 \%$ \\
\hline & gesteinsindifferente Moose & 11 Arten $=65 \%$ \\
\hline Klima: & $\begin{array}{l}\text { hygrophile Moose } \\
\text { scialophile Moose }\end{array}$ & $\begin{aligned} 10 \text { Arten } & =59 \% \\
4 \text { Arten } & =23 \%\end{aligned}$ \\
\hline
\end{tabular}

Es ist auffallend, daß sich die Höhlenmoosflorula aus Arten zusammensetzt, die zur Hauptsache gesteinsindifferent und hygrophil sind. Somit ist in erster Linie die hohe Luftfeuchtigkeit der Höhle der maßgebende Faktor, der die Zusammensetzung und Selektion der Höhlenmoosflorula bestimmt.

Sehr übereinstimmende Resultate haben wir beim Vergleich anderer bis heute bekannten europäischen Höhlenmoosfloren erhalten.

\section{Zusammenstellung der bis heute bekannten Lampen-Moosfloren europäischer Schauhöhlen}

3.1. Qualitative Zusammensetzung der Lampen-Moosflora aus 19 Schauhöhlen Europas

Es wurden folgende 19 Höhlen berücksichtigt:

1. Beatushöhle (Schweiz)

2. Grotte de Han (Belgien)

3. Charlottenhöhle (Schwäbische Alb)

\section{Alb (Deutschland)}

5. Recken- und Deckenhöhle (Westfalen)

6. Punkva- und Jaskine-Slobody-Höhlen (Tschechoslowakei)

7. Abaligeter-Höhle und weitere 3 Höhlen aus Ungarn
(Lüdy 1924;

eigene Resultate)

(Coûteaux 1956;

Duvigneaud 1939)

(Mahler 1960)

(Vogellehner

1963a)

(Koppe 1961)

(Boros 1964)

(Boros 1964) 
Tabelle 8

Die Lampen-Moosflorula von europäischen Höhlen

(Die Numerierung entspricht der obigen Höhlenliste)

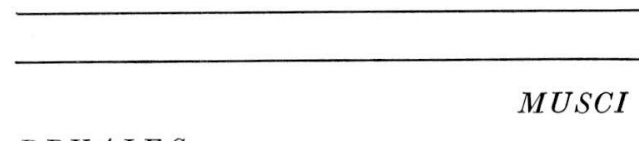

BRYALES

STEGOCARPI

\section{Weisiaceae}

Eucladium verticillatum Br. Eur.

Weisia (= Hymenostylium) microstomum Mül.

Weisia rutilans Lindb.

Hymenostylium curvirostre Lindb.

Gymnostomum rupestre Schleich.

Fissidentaceae

Fissidens taxifolius Hedw.

Fissidens cristatus Wils. ( $F$ F. decipiens DeNot)

Fissidens bryoides Hedw.

Fissidens pusillus Wils.

Fissidens exilis Hedw.

Seligeriaceae

Seligeria doniana G. Müll.

Pottiaceae

Tortella tortuosa Limpr.

Pottia truncatula Lindb.

Trichostomum mutabile Bruch.

Tortula muralis Hedw.

Barbula cylindrica (Tayl.) Schimp.

Barbula glauca (Ryan) Möll.

Barbula rigidula (Hedw.) Mitt.

\section{Encalyptaceae}

Encalypta contorta Lind. (= E. streptocarpa Hedw.)

\section{Bryaceae}

Bryum sp.

Bryum capillare L.

Bryoerythrophyllum recuroirostrum Chen.

Leptobryum pyriforme Schimp.

Mniobryum albicans Limpr.

Pohlia cruda Lindb. (=Webera cruda Bruch)

\section{Mniaceae}

Mnium punctatum Hedw.

Mnium affine Bland.

Mnium stellare Reich

Mnium subglobosum Br. Eur. (= M. pseudopunctatum Bruch. \& Schimp.)

\section{Thamniaceae}

Thamnium alopecurum Br. Eur.

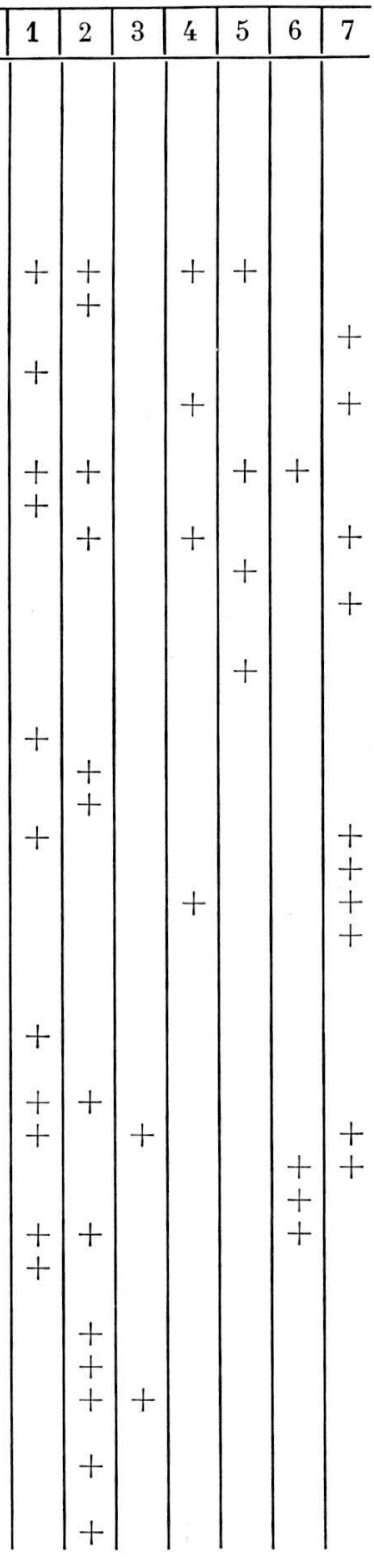


Tabelle 8 (Fortsetzung)

\begin{tabular}{|c|c|c|c|c|c|c|c|}
\hline & 1 & 2 & 3 & 4 & 5 & 6 & 7 \\
\hline CLE I STOCAR P I & & & & & & & \\
\hline Phascaceae & & & & & & & \\
\hline Phascum acaulon L. (= Ph. cuspidatum Schreb.) & & & + & & & & \\
\hline PLEUROCARPI & & & & & & & \\
\hline Neckeraceae & & & & & & & \\
\hline Neckera complanata Hüben. & & & + & & & & \\
\hline Leskeaceae & & & & & & & \\
\hline Anomodon longifolius Bruch. & & & & + & & & \\
\hline Amblystegiaceae & & & & & & & \\
\hline Amblystegium juratzkanum Schimp. & + & + & & + & & & \\
\hline Amblystegium varium Lindb. & & + & & + & & + & \\
\hline Amblystegium serpens Br. Eur. & & & & + & & & + \\
\hline Amblystegium sprucei Br. Eur. & & & & + & & & \\
\hline $\begin{array}{l}\text { Chrysohypnum (= Campylium) sommerfeldti Roth } \\
\text { Brachyteciaceae }\end{array}$ & & + & & + & & & \\
\hline Brachytecium salebrosum Br.Eur. & + & & & & & & \\
\hline Brachytecium selutinum Br. Eur. & & & + & + & & & \\
\hline Brachytecium rioulare Br. Eur. & & & + & & & & \\
\hline Brachytecium rutabulum Br. Eur. & & & + & & & & \\
\hline Rhyncostegium murale Br. Eur. & + & + & & + & & & \\
\hline $\begin{array}{l}\text { Rhyncostegiella algiriana Broth. (= Rhynco- } \\
\text { stegium tenellum Br. Eur.) }\end{array}$ & & & & + & & & \\
\hline Eurynchium schwartzi Hobkirch (=E. prae- & & & & & & & \\
\hline $\begin{array}{l}\text { (ongum Auct.) } \\
\text { Eurunchium stockesi Br Eur }\end{array}$ & + & + & & & + & & \\
\hline Eurynchium rusciforme Milde (= Rhyncostegium & & + & & & & & \\
\hline rusciforme [Neck.] B. S.) & + & & & & & & \\
\hline Plagiotheciaceae & & & & & & & \\
\hline Plagiothecium (Taxiphyllum) depressum Dix. & + & + & & + & & & + \\
\hline Hypnaceae & & & & & & & \\
\hline Ctenidium molluscum Mitten. & & & + & & & & \\
\hline HEPATICAE & & & & & & & \\
\hline$J U N G E R M A N N A L E S$ & & & & & & & \\
\hline Haplolaeneae & & & & & & & \\
\hline Pellia fabbroniana Raddi ( $=P$. calycina N.v.E. $)$ & & & & & & & + \\
\hline Pellia epiphylla Lindb. & + & & & & & & \\
\hline Pellia sp. & & + & & & & & \\
\hline Epigoniantheae & & & & & & & \\
\hline Lophozia sp. & & + & & & & & \\
\hline Metzgeriaceae & & & & & & & \\
\hline Metzgera sp. & & + & & & & & \\
\hline
\end{tabular}

\section{2. Ökologie der Höhlenmoose}

Folgende Tab. 9 beruht auf Angaben von Burck (1947) und von Lorch (1913). Man berücksichtigt Substrat und klimatologische Faktoren. 
Tabelle 9

Ökologie der Höhlenmoose

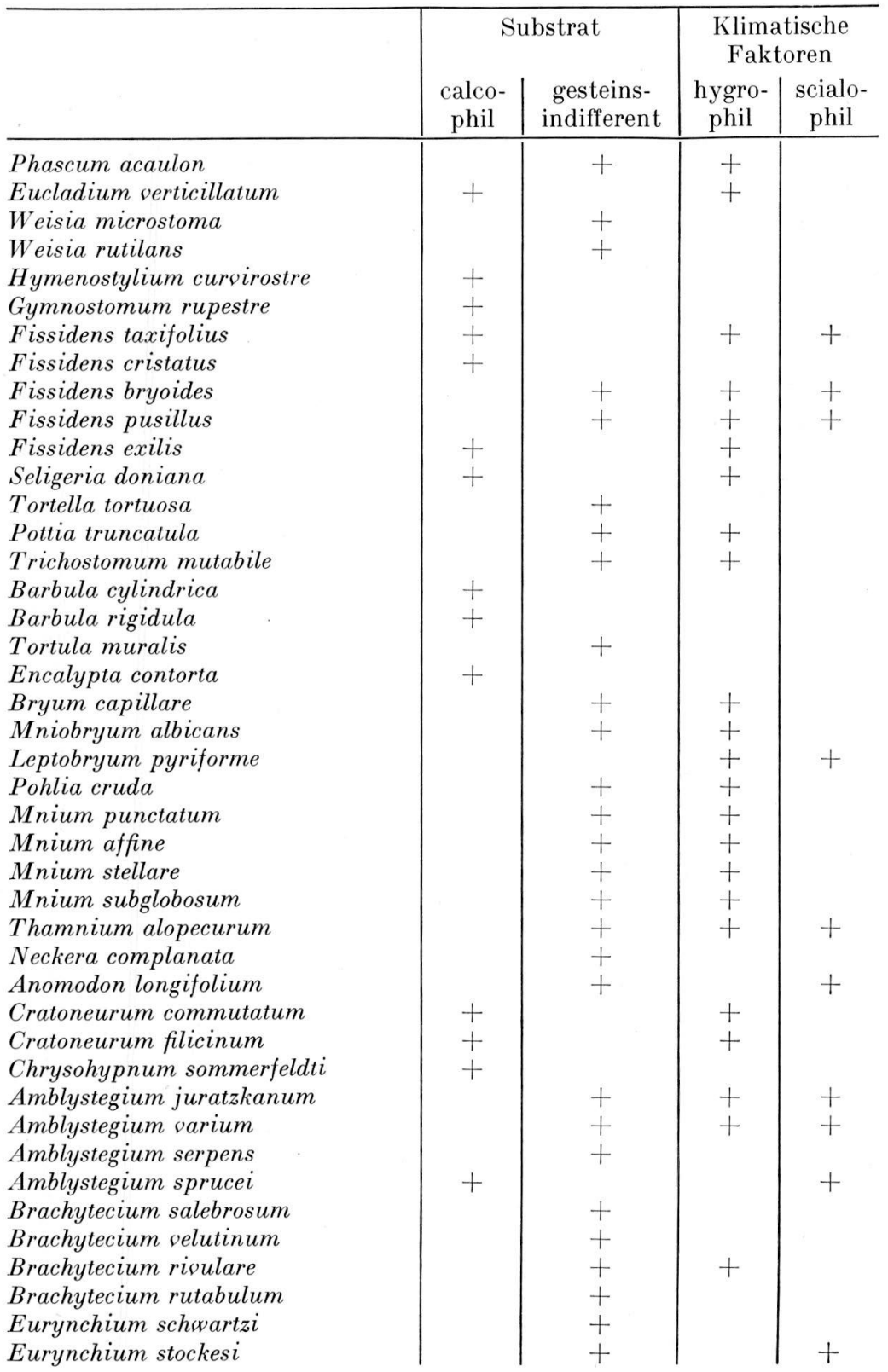


Tabelle 9 (Fortsetzung)

\begin{tabular}{|c|c|c|c|c|}
\hline & \multicolumn{2}{|c|}{ Substrat } & \multicolumn{2}{|c|}{$\begin{array}{c}\text { Klimatische } \\
\text { Faktoren }\end{array}$} \\
\hline & $\begin{array}{l}\text { calco- } \\
\text { phil }\end{array}$ & $\begin{array}{l}\text { gesteins- } \\
\text { indifferent }\end{array}$ & $\begin{array}{l}\text { hygro- } \\
\text { phil }\end{array}$ & $\begin{array}{l}\text { scialo- } \\
\text { phil }\end{array}$ \\
\hline $\begin{array}{l}\text { Eurynchium rusciforme } \\
\text { Rhyncostegium murale } \\
\text { Rhyncostegiella algiriana } \\
\text { Plagiothecium depressum } \\
\text { Ctenidium molluscum }\end{array}$ & + & $\begin{array}{l}+ \\
+ \\
+\end{array}$ & $\begin{array}{l}+ \\
+ \\
+ \\
+\end{array}$ & $\begin{array}{l}+ \\
+\end{array}$ \\
\hline
\end{tabular}

Der prozentuale Anteil der vier ökologischen Gruppen (48 Arten) ist:

\author{
Calcophile Arten \\ Gesteinsindifferente Arten \\ Hygrophile Arten \\ Scialophile Arten
}

$$
\begin{aligned}
& 16 \text { Arten }=33 \% \\
& 31 \text { Arten }=62 \% \\
& 27 \text { Arten }=56 \% \\
& 13 \text { Arten }=27 \%
\end{aligned}
$$

Wie dies für die Moosflorula der Beatushöhle festgestellt wurde, besteht der größte Anteil der Höhlen-Lampen-Moosflora aus gesteinsindifferenten und hygrophilen Arten; calcophile und scialophile Moose sind bedeutend schwächer vertreten.

\subsection{Die typischen Vertreter der Höhlen- Lampen-Moosflorula}

Neben vereinzelten Funden kommen gewisse Moosarten mit einer solchen Häufigkeit und Konstanz vor, daß man sie als typische Vertreter des Lampen-Biotops bezeichnen kann. Es handelt sich um folgende 10 Arten, die mindestens aus 3 der 7 Höhlen bzw. Höhlengruppen erwähnt wurden (vgl. Tab. 8).

Tabelle 10

Die typischen Höhlenmoose des Lampen-Biotops

Eucladium verticillatum

Fissidens taxifolius

Fissidens bryoides

Mniobryum albicans

Mnium capillare
Eurynchium schwartzi Rhyncostegium murale Plagiothecium depressum Amblystegium juratzkanum Amblystegium varium 
Die ökologische Verteilung dieser typischen Höhlenmoose ist folgende:

\section{Tabelle 11}

Ökologische Verteilung der typischen Höhlenmoose des Lampen-Biotops

Calcophile Arten
Gesteinsindifferente Arten
Hygrophile Arten
Scialophile Arten

Calcophile Arten

Hygrophile Arten
2 Arten $=20 \%$
8 Arten $=80 \%$
9 Arten $=90 \%$
6 Arten $=60 \%$

Somit lassen sich die typischen Höhlenmoose des Lampen-Biotops als hygrophile und gesteinsindifferente Arten charakterisieren.

\section{ZUSAMMENFASSUNG}

Es wird über die Lampen-Moosflora der Beatushöhle berichtet (Tab. 3). Ein statistischer Vergleich mit Lampen-Moosfloren aus 18 anderen europäischen Höhlen zeigt, daß die Zusammensetzung und die Selektion der Lampen-Moosflora überwiegend durch Hygrophilie und Gesteinsindifferenz bedingt sind (Tab. 9). 10 Arten können als typische Vertreter der LampenMoosflora bezeichnet werden.

\section{RÉSUMÉ}

On analyse la florule bryologique des lampes de la grotte St. Béat (Tab.3). Une comparaison statistique des florules des lampes d'autres 18 grottes européennes montre que la composition et la séléction de ces florules sont détérminées principalement par l'hygrophilie et l'indifférence au substrat (Tab. 9). 10 espèces peuvent être définies comme représentants typiques de la florule bryologique des lampes de grotte aménagées (Tab. 10).

\section{Nachtrag}

Weitere Angaben über die Lampen-Moosflora finden sich in: K. VERSeghy: Die Pflanzenwelt der Höhlen bei Lillafüred, Int. J. Spel. (1965) 1 (4), 553-560.

Es wurden aus den Forras- und Istvàn-Höhlen folgende Moosarten gesammelt:

Eucladium verticillatum Br. Eur. und seine var. angustifolia, Gymnostomum rupestre Schleich. und seine fa. ramosissima, Fissidens taxifolius Hedw. und minutulus Sull., Tortula muralis Hedw. und seine var. aestisa Brid., Barbula fallax Hedw. und unguiculata Hedw., Bryum caespiticium L., Bryoerythrophyllum recurvirostrum Chen., 
Pohlia sp.,

Funaria hygrometrica Sibth.,

Amblystegium riparium Br. Eur. und serpens Br. Eur.,

Brachytecium velutinum Br. Eur.,

Rhyncostegium murale Br. Eur.,

Eurynchium schwartzi Hobkirch,

Pellia fabbroniana Raddi.

\section{TERAT UR}

Boros, A. (1964) - Über die Moose, die unter dem Einfluß der elektrischen Beleuchtung in das Innere der Höhlen in Ungarn und in der Tschechoslowakei eindringen. Internat. J. Spel. $1(1-2)$ : 45-46.

Burck, O. (1947) - Die Laubmoose Mitteleuropas. Abhdlg. Senckenberg. Nat.forsch. Ges. (477): 5-198.

Coûteaux, M. (1956) - Le milieu de la flore et de la végétation des grottes de Han. Rass. spel. ital. 8 (3-4): 155-182.

Duvigneaud, P. - Les populations végétales des grottes de Han. Ass. franç. pour l'avancement des sciences, $63^{\mathrm{e}}$ session, 939-944. Liège.

Koppe, F. (1961) - Niedere Kryptogamen und Moose in sauerländischen Höhlen. Jhft. Karst- und Höhlenkunde (2) : 245-259.

Lorсн, W. (1913) - Die Laubmoose. In: G. Lindau: Kryptogamenflora für Anfänger, Bd. 5. Berlin.

- (1914) - Die Torf- und Lebermoose. In: G. Lindau: Kryptogamenflora für Anfänger, Bd. 6. Berlin.

LÜDy, W. (1924) - Pflanzenleben der Beatushöhlen am Thunersee. Mittlg. Naturforsch. Ges. Bern XLIII.

Mahler, K. (1960) - Über die Pflanzenwelt unserer Albhöhlen. Jhft. Karstund Höhlenkunde (1): 129-136.

Morton, F. (1927) - Ökologie der assimilierenden Höhlenpflanzen. Fortschritte der nat.-wiss. Forschung 12 (3) : 155-234.

Morton, F., und Gams, H. (1925) - Höhlenpflanzen, Bd. 5 der Speläologischen Monographien. Wien.

Stammler, M. (1904) - Der heilige Beatus, seine Höhle und sein Grab. Bern.

Vogellehner, D. (1963a) - Zur Pflanzenwelt um die Lampen in einigen Schauhöhlen der Schwäbischen Alb. Jhft. Karst- und Höhlenkunde (4) : 229-244.

- (1963b) - Elektrisch beleuchtete Schauhöhlen, ein neuer pflanzlicher Lebensraum. Mittlg. Vbd. dtsch. Höhlen- und Karstforscher 9 (2): $38-39$. 